\title{
IMPLEMENTATION AND VALIDATION OF THE HARMONIC BALANCE METHOD FOR TEMPORALLY PERIODIC NON-LINEAR FLOWS
}

\author{
Hrvoje Jasak \\ Department of Energy, Power Engineering \\ and Environment \\ Faculty of Mechanical Engineering and \\ Naval Architecture \\ University of Zagreb \\ Zagreb 10 0000, Croatia \\ Email: hrvoje.jasak@fsb.hr
}

\author{
Gregor Cvijetić \\ Department of Energy, Power Engineering \\ and Environment \\ Faculty of Mechanical Engineering and \\ Naval Architecture \\ University of Zagreb \\ Zagreb 10 0000, Croatia \\ Email: gregor.cvijetic@gmail.com
}

\begin{abstract}
An efficient method for tackling non-linear, temporallyperiodic incompressible flows is presented in this paper. Assuming temporally fully periodic flow, Harmonic Balance method deploys Fourier transformation in order to formulate transient problem as a multiple quasi-steady state problems. The method is implemented in OpenFOAM and developed for a general transport equation and incompressible Navier-Stokes equations. Validation is presented on three test cases: oscillating scalar case for scalar transport validation, a flow around a $2 D$ NACA airfoil and a 3D Onera M6 wing for turbulent incompressible Navier-Stokes validation. For all test cases Harmonic Balance results are compared to transient simulation results. Verification of the model is performed by changing the number of harmonics for all test cases.
\end{abstract}

\section{NOMENCLATURE}

$\mathscr{Q}$ dimensionless passive scalar in time domain

$\mathscr{R}$ convection-diffusion transport operator for a passive scalar in time domain

$t$ time, $\mathrm{s}$

u velocity field, $\mathrm{m} / \mathrm{s}$

$\gamma \quad$ diffusion coefficient, $\mathrm{m}^{2} / \mathrm{s}$
$S_{\mathscr{Q}} \quad$ source terms for passive scalar, $1 / \mathrm{s}$

$\omega$ base radian frequency, $\mathrm{rad} / \mathrm{s}$

$\underline{A}$ discrete Fourier expansion matrix

$\overline{\bar{Q}} \quad$ vector of Fourier harmonics for $\mathscr{Q}$

$\underline{R} \quad$ vector of Fourier harmonics for $\mathscr{R}$

$\mathscr{Q} \quad$ vector of discrete time instant values for $\mathscr{Q}$

$\mathscr{R} \quad$ vector of discrete time instant values for $\mathscr{R}$

$T$ base period, $\mathrm{s}$

$\underline{\underline{E}} \quad$ forward DFT matrix

$\underline{\bar{E}}^{-1}$ backward (inverse) DFT matrix

$\bar{P}_{i-j}$ coupling coefficient for $t_{i}$ and $t_{j}$ time instants

$v \quad$ kinematic viscosity, $\mathrm{m}^{2} / \mathrm{s}$

$\rho$ density, $\mathrm{kg} / \mathrm{m}^{3}$

$p$ pressure, $\mathrm{Pa}$

$a_{P}$ diagonal coefficient in the discretised momentum equation, $1 / \mathrm{s}$

H flux operator, $\mathrm{kg} /\left(\mathrm{m}^{2} \mathrm{~s}^{2}\right)$

$f \quad$ base frequency, $\mathrm{Hz}$

$A, B$ wave amplitudes

$\phi \quad$ phase shift, $\mathrm{s}$

Subscripts

$S \quad$ sine part

C cosine part

$i$ harmonic index

$t_{j} \quad$ discrete time instant 


\section{INTRODUCTION}

Periodic flows are common in scientific and industrial studies such as rotating machinery, wave-like phenomena, various flows induced by periodic boundary conditions and different periodic body motions (wing oscillations, moving valves, etc.). For such problems transient simulations are mostly used, yielding long CPU time. In order to obtain periodic results and neglect initial transients, several periods need to be simulated. The need for reducing CPU time, but still preserving periodic flow features motivated the development of new methods. Harmonic Balance [1,2] is such a method, developed specially for periodic problems with prescribed harmonic motion.

As opposed to conventional steady state methods, the benefit of Harmonic Balance is the ability of capturing transient flow features, but at a cost of longer CPU time. However, compared to conventional transient simulation Harmonic Balance offers a significant CPU time reduction [3] with comparable accuracy. Although it was initially developed as a periodic boundary condition [4], He and Ning [5] extended its application to solving the two-dimensional Navier-Stokes equations and presented the efficiency improvement compared to nonlinear time-marching methods. Recently, Harmonic Balance method has been extensively developed in numerous application areas. Other than oscillating airfoils and wings presented by Dufour et al. [6] and Thomas et al. [7, 8], also aeroelastic flutter and limit cycle oscillations [9, 10] were the topic of many research. Hall et al. [11] used complex geometries such as turbines to extend and demonstrate the Harmonic Balance capabilities, which was later used by Nadarajah et al. [12] in performing shape design optimisation. Multiple frequency approach is also used by Gopinath et al. [13] and Guédeney et al. [14] for simulations of multistage turbines where rotor frequency changes in each stage due to different number of blades.

This paper presents the mathematical model of the Harmonic Balance method, as well as validation and verification of the method. Hall et al. [15] proposed 3 forms of Harmonic Balance, of which time-spectral form of Harmonic Balance is used here. The method is based on Fourier series expansion, suggesting that the frequency of the motion should be known in advance, while number of harmonics is a user-chosen input parameter. Number of harmonics is dictated by several important elements:

- The number of equations to be solved depends on the number of harmonics: for $n$ harmonics $2 n+1$ equations are solved. Utilizing Harmonic Balance treatment, equations to be solved become coupled for each variable containing the time-derivative term.

- Due to the temporally-coupled $2 n+1$ equations, larger number of equations slow down the convergence beyond the simple increase in the number of unknowns.

- The number of harmonics dictates the accuracy, as higher order effects are neglected.

The paper is organised as follows. Mathematical model of the Harmonic Balance method is briefly presented, including scalar transport equation and incompressible Navier-Stokes equations. Finite volume implementation of the governing equations is given in section 3 , followed by the turbulence modelling and mesh motion. Section 6 deals with validation of the Harmonic Balance method, which is performed with three test cases. First test case considers validation of scalar transport equation in Harmonic Balance form. Rectangular domain with oscillating scalar on the inlet boundary is simulated, imposing 2 types of periodic phenomena: harmonic and steep signals. Convergence study with variable number of harmonics is also performed. Validation of Navier-Stokes equations in Harmonic Balance form with turbulence models is performed using a 2D airfoil and a 3D wing test case. Pitching NACA 2412 airfoil is simulated for high and low Reynolds numbers and compared to the transient simulation. For the 3D case, only one Reynolds number is presented, using Onera M6 pitching wing. A global overview of the results is given with a short conclusion.

\section{MATHEMATICAL MODEL}

An overview of the mathematical model of the Harmonic Balance (HB) method is presented in this section. HB treatment, transforming the time-derivative term into a source term, is done on scalar transport equation and then extended to incompressible flow equations. Mathematical model presented here is general and valid for any number of harmonics. A full derivation can be found in [3].

\section{Passive Scalar Transport}

Convection-diffusion equation for passive scalar transport of scalar $\mathscr{Q}$ reads:

$$
\frac{\partial \mathscr{Q}}{\partial t}+\mathscr{R}=0
$$

where $\mathscr{R}$ stands for convection, diffusion and source/sink terms:

$$
\mathscr{R}=\nabla \cdot(\mathbf{u} \mathscr{Q})-\nabla \cdot(\gamma \nabla \mathscr{Q})-S_{\mathscr{Q}},
$$

$\mathbf{u}$ is the transport velocity and $\gamma$ is diffusivity. Expanding $\mathscr{Q}$ into Fourier series with $n$ harmonics reads:

$$
\mathscr{Q}(t)=Q_{0}+\sum_{i=1}^{n} Q_{S_{i}} \sin (i \omega t)+Q_{C_{i}} \cos (i \omega t) .
$$

Scripture characters, $\mathscr{Q}$, are used to denote time domain variables, while $Q$ denotes frequency domain field. The Fourier ex- 
pansion for $\mathscr{R}$ is analogous to the one in Eqn. (3), with $Q$ substituted with $R$. Inserting Eqn. (3) into the transport equation, Eqn. (1), yields sine, cosine and mean terms. Grouping the terms gives $2 n+1$ equations: $n$ for sine and cosine and 1 for the mean value. Thus, a HB scalar transport equation becomes a set of $2 n+1$ equations, written in matrix form:

$$
\omega \underline{\underline{A}} \underline{Q}+\underline{R}=\underline{0}
$$

where $\underline{A}$ is a $(2 n+1) \times(2 n+1)$ coefficients matrix, $Q$ and $\underline{R}$ are column matrices containing Fourier sine $Q_{S_{i}} / R_{S_{i}}$ and cosine $Q_{C_{i}} / R_{C_{i}}$ coefficients and $\omega$ is a base radian frequency.

In order to easily switch between the time and frequency domain, a matrix representation of Direct Fourier Transform (DFT) is introduced. DFT converting from the time-domain vector $\mathscr{Q}$ to the frequency domain vector $\underline{Q}$ can be written in matrix form:

$$
\underline{Q}=\underline{\underline{E}} \underline{\mathscr{Q}}
$$

where $\underline{\mathscr{Q}}$ is a discrete time-domain vector needed for unique oneto-one mapping. $\mathscr{Q}$ is defined as:

$$
\underline{\mathscr{Q}}^{T}=\left[\begin{array}{lllll}
\mathscr{Q}_{t_{1}} & \mathscr{Q}_{t_{2}} & \mathscr{Q}_{t_{3}} & \cdots & \mathscr{Q}_{t_{2 n+1}}
\end{array}\right]
$$

where $t_{i}$ stands for:

$$
t_{i}=\frac{i T}{2 n+1}, \text { for } i=1 \ldots 2 n+1
$$

Multiplying Eqn. (5) with $\underline{\underline{E}}^{-1}$ from the left, one obtains a mapping from the frequency domain to the time domain:

$$
\underline{Q}=\underline{\underline{E}}^{-1} \underline{Q}
$$

Using the forward and backward transformation matrices $\underline{E}$ and $\underline{\underline{E}}^{-1}$, the frequency domain scalar transport equation, Eqn. (4), is formulated using the time-domain vector $\underline{\mathscr{Q}}$ :

$$
\omega \underline{\underline{A}} \underline{\underline{E}} \underline{\mathscr{Q}}+\underline{\underline{E}} \underline{\mathscr{R}}=\underline{0}
$$

where the same transformation has been applied to $\underline{R}$ and $Q$. Even though equations could be solved in this form, evaluating sources and fluxes in the frequency domain is computationally expensive and inconvenient [1]. Therefore, the equation is transformed back to time domain, multiplying the Eqn. 9 with $\underline{\underline{E}}^{-1}$ from the left:

$$
\omega \underline{\underline{E}}^{-1} \underline{\underline{A}} \underline{\underline{E}} \underline{\mathscr{Q}}+\underline{\mathscr{R}}=\underline{0} .
$$

The resulting equation represents a temporally-coupled set of $2 n+1$ steady state problems. Comparing Eqn. (10) with the original scalar transport equation, Eqn. (1), two important features may be observed:

- $\mathscr{R}$ has been replaced with its discrete counterpart $\underline{\mathscr{R}}$, indicating that the solution is sought at a fixed number of discrete time instants only. The number of discrete time instants is defined with specified number of harmonics $n$, as indicated in Eqn. (6).

- Time derivative term has been replaced by terms coupling the solutions at different time steps. This is equivalent to evaluating the time derivative of a harmonic signal via $2 n+$ 1 uniformly spaced temporal snapshots, including a mean (steady) solution.

The expanded form of the coupled HB scalar transport equations may be written in a more convenient form:

$$
\begin{gathered}
\nabla \cdot\left(\mathbf{u} \mathscr{Q}_{t_{j}}\right)-\nabla \cdot\left(\gamma \nabla \mathscr{Q}_{t_{j}}\right)-S_{\mathscr{Q}_{t_{j}}}=-\frac{2 \omega}{2 n+1}\left(\sum_{i=1}^{2 n} P_{i-j} \mathscr{Q}_{t_{i}}\right) \\
\text { for } j=1 \ldots 2 n+1
\end{gathered}
$$

where $P_{i}$ is defined as:

$$
P_{i}=\sum_{k=1}^{n} k \sin (i k \omega \Delta t), \text { for } i=1 \ldots 2 n
$$

and:

$$
\Delta t=\frac{T}{2 n+1} .
$$

The coupling of solutions at different time instants $t_{j}$ is achieved through $P_{i-j}$ matrix, modelling the time derivative term as additional source terms. Hence, a single transient equation given by Eqn. (1) is transformed into a set of $2 n+1$ coupled steady state problems, Eqn. (11).

\section{Incompressible Fluid Flow}

Incompressible, turbulent, single-phase flow is modelled with the continuity and the momentum equation:

$$
\nabla \cdot \mathbf{u}=0
$$

$$
\frac{\partial \mathbf{u}}{\partial t}+\nabla \cdot(\mathbf{u u})-\nabla \cdot(v \nabla \mathbf{u})=-\frac{\nabla p}{\rho},
$$


where $v$ denotes kinematic viscosity, $\rho$ fluid density and $p$ the pressure field.

As previously presented, HB treatment transforms the time derivative term into a set of temporally-coupled source terms: leaving convection, diffusion and additional source terms in their original form. The continuity equation remains the same, as it does not contain a time-derivative term:

$$
\nabla \cdot \mathbf{u}_{t_{j}}=0 .
$$

Equation (16) suggests that the incompressible continuity equation must hold in each time instant $t_{j}$, as expected. The HB form of the momentum equation reads:

$$
\nabla \cdot\left(\mathbf{u}_{t_{j}} \mathbf{u}_{t_{j}}\right)-\nabla \cdot\left(v \nabla \mathbf{u}_{t_{j}}\right)=-\nabla p_{t_{j}}-\frac{2 \omega}{2 n+1}\left(\sum_{i=1}^{2 n} P_{i-j} \mathbf{u}_{t_{i}}\right),
$$

$$
\text { for } j=1 \ldots 2 n+1 \text {. }
$$

Equations (16) and (17) represent $2 n+1$ coupled pressurevelocity systems with enforced periodic behaviour defined using the base frequency $\omega$ and number of harmonics $n$.

\section{FINITE VOLUME IMPLEMENTATION}

This section briefly presents the Finite Volume (FV) implementation of the scalar transport equation and pressure-velocity system in the HB form. Notation used here follows the one presented by Rusche [16], denoting the implicit FV discretisation with [.]. Discretised HB scalar transport equation, Eqn. (11) reads:

$$
\left[\nabla \cdot\left(\mathbf{u} \mathscr{Q}_{t_{j}}\right)\right]-\left[\nabla \cdot\left(\gamma \nabla \mathscr{Q}_{t_{j}}\right)\right]-\left[S_{\mathscr{Q}_{t_{j}}}\right]=-\frac{2 \omega}{2 n+1}\left(\sum_{i=1}^{2 n} P_{i-j} \mathscr{Q}_{t_{i}}\right),
$$

$$
\text { for } j=1 \ldots 2 n+1 \text {, }
$$

while the discretised form of $\mathrm{HB}$ momentum and pressure equation are given by:

$$
\begin{gathered}
{\left[\nabla \cdot\left(\mathbf{u}_{t_{j}} \mathbf{u}_{t_{j}}\right)\right]-\left[\nabla \cdot\left(v \nabla \mathbf{u}_{t_{j}}\right)\right]=-\nabla p_{t_{j}}-\frac{2 \omega}{2 n+1}\left(\sum_{i=1}^{2 n} P_{i-j} \mathbf{u}_{t_{i}}\right),} \\
{\left[\nabla \cdot\left(\frac{1}{a_{P t_{j}}} \nabla p_{t_{j}}\right)\right]=\nabla \cdot\left(\frac{\mathbf{H}\left(\mathbf{u}_{t_{j}}\right)}{a_{P t_{j}}}\right),}
\end{gathered}
$$

$$
\text { for } j=1 \ldots 2 n+1 \text {. }
$$

HB pressure equation, Eqn. (20) follows from the HB continuity equation, Eqn. (16), using standard procedure for segregated solution algorithms. $a_{P t_{j}}$ is the diagonal coefficient of the momentum equation at time instant $t_{j}$ and $\mathbf{H}\left(\mathbf{u}_{t_{j}}\right)$ is the flux operator, as defined by Jasak [17].

Equations (18) to 20) show that the source terms arising from HB treatment of the time derivative term are treated explicitly. Hence, a segregated, iterative solution algorithm for successive $\mathscr{Q}_{t_{j}}$ and $\mathbf{u}_{t_{j}}$ is employed.

To resolve the pressure-velocity coupling at each time instant $t_{j}$, SIMPLE [18] algorithm is used. In addition to pressurevelocity coupling at each outer iteration, velocity fields at different time instants are coupled due to HB source terms on the right hand side of Eqn. (19). Following analogy with Gauss-Seidel iterative solution algorithm [19], $\mathscr{Q}_{t_{j}}, \mathbf{u}_{t_{j}}$ and $p_{t_{j}}$ is solved once per outer iteration during a forward sweep $(j=1 \ldots 2 n+1)$. Latest available variable is always used in source terms for other equations $(k>j)$, preventing additional memory requirements. Outer iterations are continued until convergence.

\section{TURBULENCE MODELLING}

Turbulence modelling is included in all of the presented cases. Two equation eddy-viscosity turbulence model is used: Shear Stress Transport formulation of k- $\omega$ turbulence model by Menter [20]. Analogous to scalar transport equation, the HB treatment of $k$ and $\omega$ equations yields transformation of timederivative term into a set of source terms.

\section{MESH MOTION}

In order to model pitching airfoils in HB simulations, mesh motion needs to be taken into account. In each outer iteration, all of the time steps are calculated $\left(t_{j}=j T /(2 n+1)\right.$, for $j=1 \ldots 2 n+1)$, which requires the mesh motion to be performed $2 n+1$ times per outer iteration. Due to the mesh motion, the mesh motion flux needs to be updated. Mesh flux calculation is performed according to Space Conservation Law [21] using standard OpenFOAM tools. During each outer iteration, before calculation of the $n$-th harmonic variables, time interval is set to $\Delta t=T /(2 n+1)$. Time interval manipulation yields correct mesh motion for each harmonic. This way existing OpenFOAM mesh library can be used, regardless of simulation setup (time step, etc.). Mesh flux is calculated using linear function of swept volume $V_{\text {swept }}=\Delta t \sum_{\text {faces }}\left(\mathbf{u}_{\text {face }} . \mathbf{n} d S\right)$, which is a good approximation for small amplitude rotations. For cases such as turbomachinery, where mesh motion between successive HB time instants is large and not linear, the presented approach may not be sufficiently accurate. Let us assume the rotation of a point at 
radius $r$ rotating $\pi$ radians. The trajectory calculated using presented approach would evaluate as $2 r$ instead of $\pi r$, therefore such problems require different treatment.

\section{VALIDATION}

This section presents the validation and verification of $\mathrm{HB}$ variants of scalar transport and Navier-Stokes equations. Four periodic scalar transport test cases are presented using the oscillating boundary condition at the inlet. In first two test cases harmonic signals are imposed, while the last two deal with steep signals: such test cases demonstrate the robustness of the method. A convergence study regarding different number of harmonics is also performed to verify the implemented method. In the second part of validation, HB form of Navier-Stokes equations is considered. Two test cases are used: pitching 2D NACA 2412 airfoil and pitching 3D Onera M6 wing. NACA 2412 simulation is performed for high and low Reynolds number with turbulence modelling included. Onera M6 wing is simulated using one Re number. Convergence study concerning variable number of harmonics is presented. All the results are compared to transient simulation.

\section{Scalar Transport: Validation and Verification}

Scalar transport validation is performed on a $2-D$ rectangular domain, uniformly discretised with 6633 hexahedral cells. The domain size is $10 \times 7 \mathrm{~m}$. Uniform velocity of $\mathbf{u}=10 \mathrm{~m} / \mathrm{s}$ is prescribed, with the diffusion coefficient of $\gamma=1.5 \cdot 10^{-5} \mathrm{~m}^{2} / \mathrm{s}$. Boundary conditions at the inlet are prescribed for each test case differently. The HB and transient simulation results are compared along the centerline of the domain $(x \in[0 \mathrm{~m}, 10 \mathrm{~m}], y=0$ m) for all test cases.

Single Sine Wave. The first test case is modelled prescribing the boundary condition corresponding to a sine wave:

$$
\mathscr{Q}(t)=A \sin (2 \pi f t),
$$

with $A=5$ being the amplitude of the sine wave and $f=2 \mathrm{~Hz}$ is the frequency. In $\mathrm{HB}$ simulation, each time instant $t_{j}$ is prescribed with the corresponding value $\mathscr{Q}_{t_{j}}=A \sin \left(2 \pi f t_{j}\right)$. Zero gradient boundary condition is used for other boundaries. Since sine wave is imposed, only one harmonic is used, $n=1$. Figure 1 presents comparison of $\mathrm{HB}$ results to transient simulation: a visualisation of wave-like scalar propagating through the domain and $\mathrm{HB} /$ transient simulation comparison at different time instants.

Sine-Cosine Wave Combination. The second test case is modelled prescribing the boundary conditions corre-

\section{TWO SINE WAVES PROPAGATING}
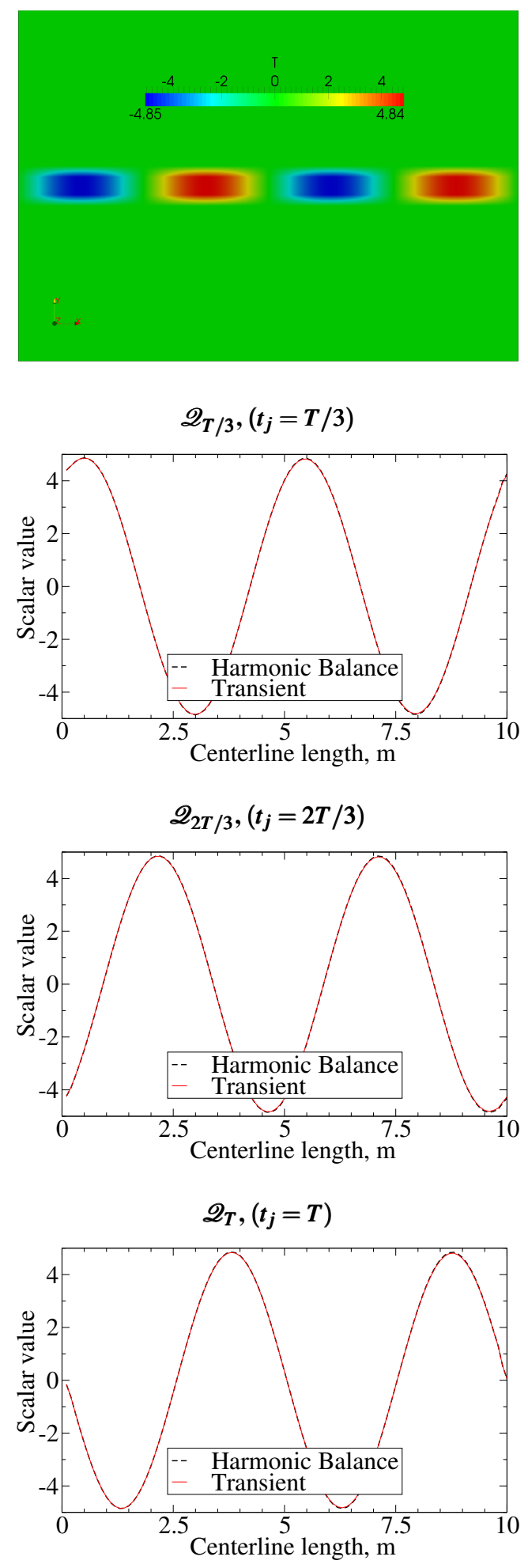

FIGURE 1: HB AND TRANSIENT SIMULATION COMPARISON FOR SINE WAVE. 
sponding to a linear combination of sine and cosine wave:

$$
\mathscr{Q}(t)=A \sin (2 \pi f(t-\phi))+B \cos (2 \pi 2 f(t-\phi)),
$$

where $A=3$ is the amplitude of the sine wave and $B=5$ is the amplitude of the cosine wave. Base frequency is $f=1 \mathrm{~Hz}$ and $\phi=0.35$ is arbitrary phase shift used to position the wave to zero value at $t=0$. Due to the different frequency of sine and cosine terms $f_{\cos }=2 f_{\sin }, n=2$ harmonics are used. Other boundary conditions are defined as in the first test case. Comparison of HB results to transient simulation at different time instants is presented in Fig. 2

Single Steep Wave. In order to demonstrate the robustness and capability of the method, steep wave test cases are presented. Steep waves are usually numerically more demanding and represent a challenge to numerical algorithms. Excitation at the inlet boundary is depicted in Fig. 3 at the top, with the frequency of $f=1 \mathrm{~Hz}$. The comparison of wave profiles at the end of a period for varying number of harmonics $(n=3,5,7)$ is presented in Fig. 3 at the bottom. Increasing the number of harmonics used, the HB solution converges to the transient one. The results for different time instants exhibit similar behaviour and are not presented.

Complex Steep Wave. In the final scalar transport test case, a more complex steep wave is simulated. $n=3,5,7$ and 10 harmonics are used and compared with transient simulation. Top most figure in Fig. 4 presents a complex steep wave excitation at the inlet boundary. The HB and transient simulation comparison at the end of a period is presented in the middle figure, while the solution convergence with increased number of harmonics is presented in the bottom figure.

The bottom most figure in Fig. 4 shows the relative error between transient solution and the HB solution with $n=3,5,7$ and 10 harmonics. Bars at the bottom of the graph represent the modulus of the relative error (at a different scale). Bars are used to show the small difference between 7 and 10 harmonics results. Green bars denote 10 harmonics solution, while red bars denote 7 harmonics. It is important to notice that HB simulation with 7 harmonics involves solving 15 coupled equations, while 10 harmonics involves 21 coupled equation. It is an unnecessary substantial increase in terms of memory and CPU cost, with insignificant differences between results. The mesh resolution also dictates the highest relevant harmonic. For coarse mesh simulations there will be no difference between two harmonics if disturbances they capture are smaller than disturbances the mesh is able to resolve.
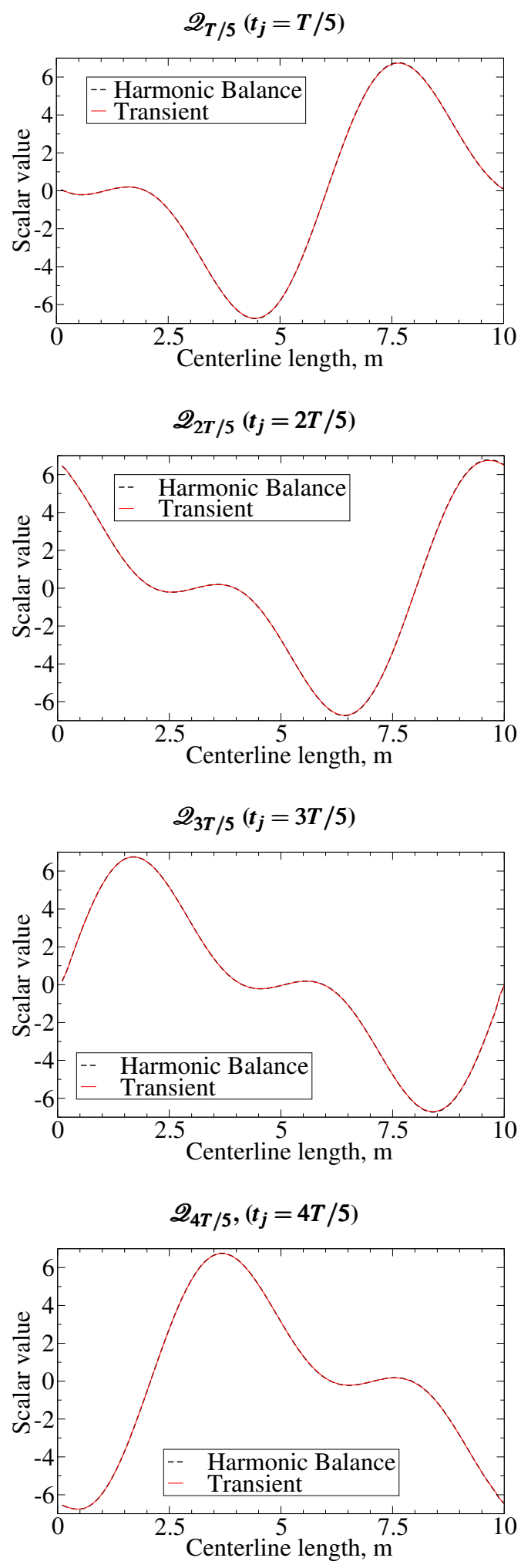

FIGURE 2: HB AND TRANSIENT SIMULATION COMPARISON FOR A SINE-COSINE WAVE COMBINATION.

Copyright (c) 2016 by ASME 
Steep wave imposed at the inlet boundary

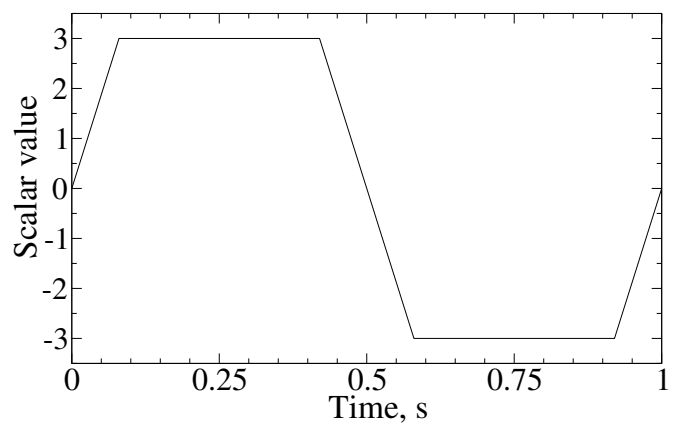

$\mathscr{Q}_{T}\left(t_{j}=T\right)$ for varying number of harmonics

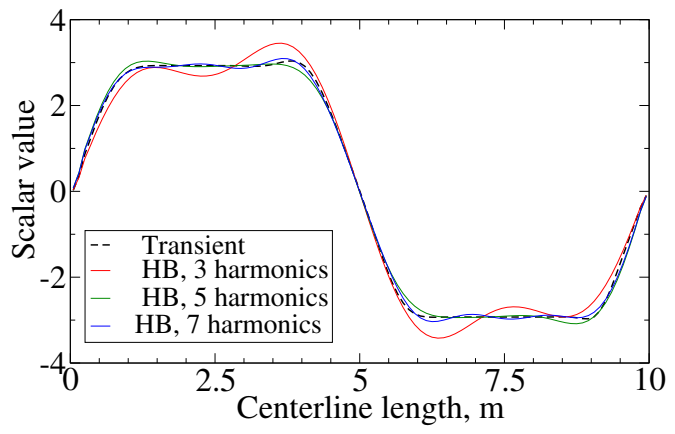

FIGURE 3: HB AND TRANSIENT SOLUTION COMPARISON FOR A STEEP WAVE.

\section{Incompressible Flow Validation and Verification}

This section deals with validation of incompressible NavierStokes equations in HB form. Two test cases are used: NACA 2412 airfoil and Onera M6 wing. NACA 2412 is a 2D case presented in high and low Reynolds number variants. The results are obtained for different number of harmonics and compared with transient simulation. Onera M6 test case is a 3D pitching wing, simulated with 1 and 3 harmonics. All of the presented cases include turbulence models and moving mesh effects. The two-equation Menter's $k-\omega S S T$ model is used.

Pitching NACA 2412, low $R$ e. NACA 2412 is a 2D validation case with circular domain of diameter $d=13 \mathrm{~m}$ and 6060 hexahedral cells, depicted in Fig. 5. Airfoil chord length is $1 \mathrm{~m}$ and pitching axis is located at the chord line at one third distance from the trailing edge. Pitching period is set to $10 \mathrm{~s}$ and the pitching angle follows the sine rule: $\theta=3 \sin (2 \pi f t)$ which is modelled by rotating the mesh.

For the low $R e$ case inlet velocity is uniform and constant, $\mathbf{u}=1 \mathrm{~m} / \mathrm{s}$ and kinematic viscosity is set to $v=1.5 \cdot 10^{-5} \mathrm{~m}^{2} / \mathrm{s}$ which yields $R e=66667$. Figure 6 and 7 present pressure contours at the airfoil surface. Expanded airfoil cells are used (hor-

\section{Complex steep wave imposed at the inlet}

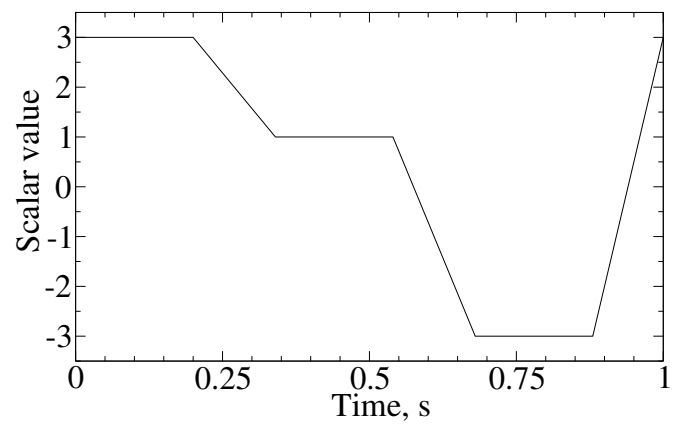

$\mathscr{Q}_{T}\left(t_{j}=T\right)$ for varying number of harmonics

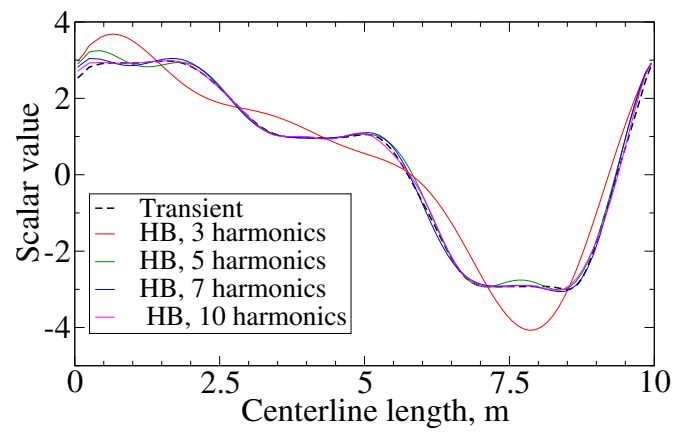

\section{Errors between HB and transient simulation}

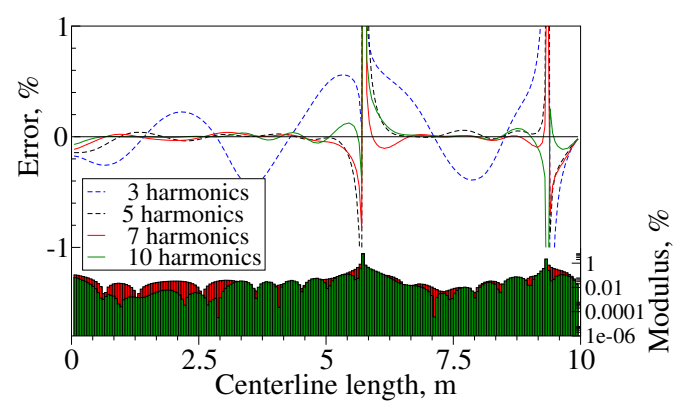

\section{FIGURE 4: HB AND TRANSIENT SOLUTION COMPARISON FOR A COMPLEX STEEP WAVE.}

izontal axis) in order to be able to compare result in each cell. The pressure peak at the middle presents the leading edge stagnation point while horizontal axis values 0 and 100 stand for trailing edge. This notation will be used in all of the following cases, with variation in cell number. Pressure contours at $t=T / 4, t=2 T / 4, t=3 T / 4$ and $t=T$ are compared for solutions obtained using 1,3 and 5 harmonics with transient results. HB pressure contour follows the transient result closely, showing that periodic motion is fully captured. Peak pressures show good agreement in all of the time steps. Comparable accuracy can be 


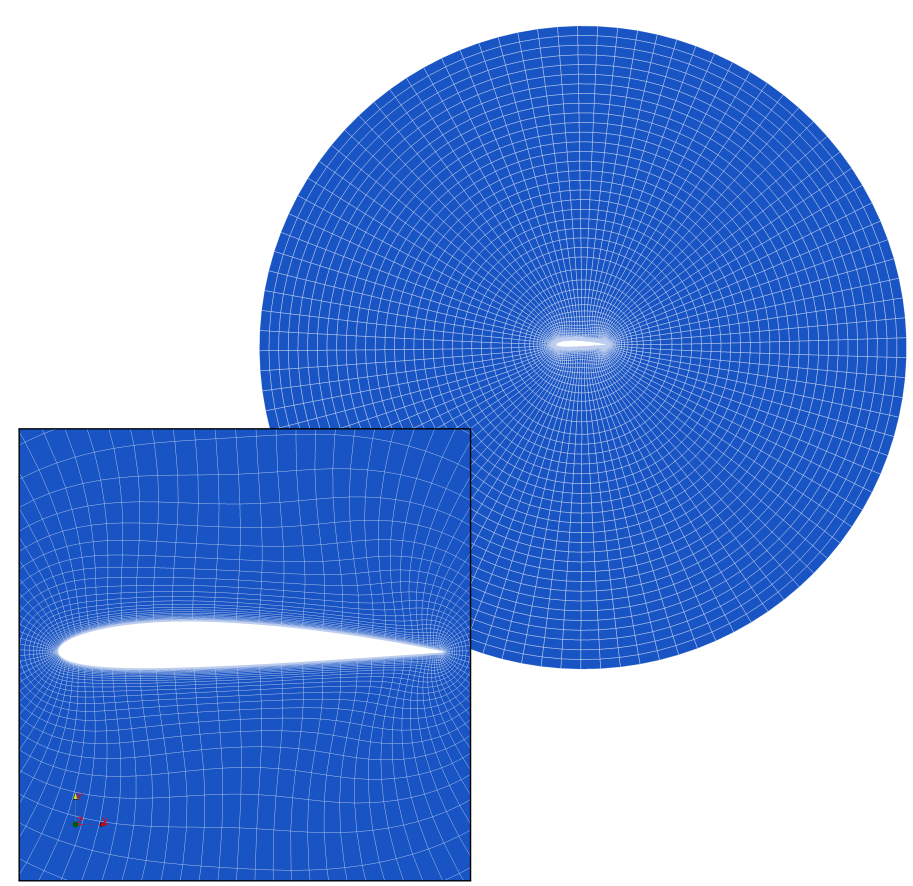

FIGURE 5: NACA 2412 DOMAIN.

noticed between solutions for 1 harmonic and for 3 or 5 harmonics. The discrepancy between 1 harmonic solution and transient solution is most noticeable at the pressure peak for $t=2 T / 4$ and $t=3 T / 4$. The solution is converging towards transient result with increased number of harmonics as indicated by Fig. 7 . The number of harmonics $n=3$ is sufficient for this case, as the difference between 3 and 5 harmonics solution is insignificant.

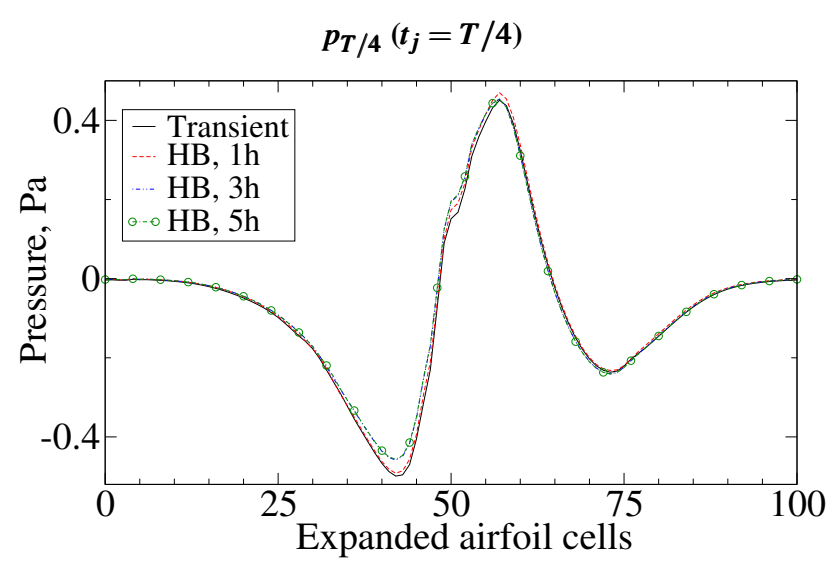

FIGURE 6: COMPARISON OF PRESSURE CONTOURS AROUND THE AIRFOIL AT LOW $R e$ FOR $t_{j}=T / 4$.
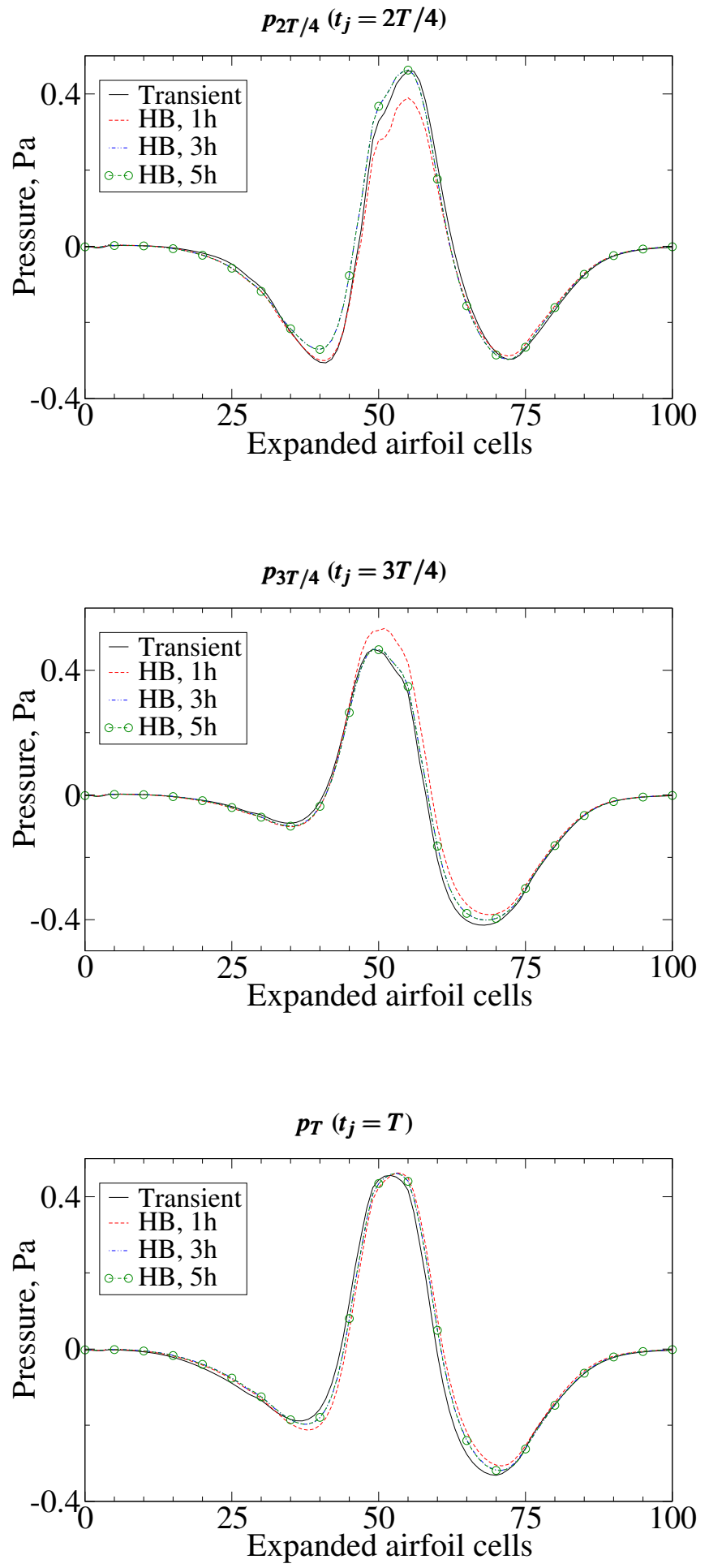

FIGURE 7: PRESSURE CONTOURS AROUND THE AIRFOIL AT LOW $\operatorname{Re}$ FOR $t_{j}=2 T / 4,3 T / 4$ and $T$. 
Authors believe that the linear calculation of mesh motion fluxes is the cause of slight discrepancy visible as an offset between the pressure peak for $t=T$. The offset caused by linear approximation of mesh flux diminishes by increasing the flow speed, which is presented in the NACA 2412 high Re case.

Pitching NACA 2412, high Re. Inlet velocity of the high $R e$ NACA 2412 pitching airfoil is set to $\mathbf{u}=15 \mathrm{~m} / \mathrm{s}$, with kinematic viscosity $v=1.5 \cdot 10^{-5} \mathrm{~m}^{2} / \mathrm{s}$, which yields $R e=$ 1000000 . HB simulation is performed using 1,3 and 5 harmonics and compared to transient simulation.

Results presented in Fig. 8 and 9 show the comparison of $\mathrm{HB}$ and transient simulation. Pressure contours around the airfoil are presented for $t=T / 4, t=2 T / 4, t=3 T / 4$ and $t=T$. Periodic motion is fully captured and pressure peak is successfully resolved without any major discrepancies. The convergence with increasing number of harmonics is achieved, with largest difference in transition from 1 to 3 harmonics. The solution for 1 harmonic differs from the transient solution significantly, while 3 harmonics solution agrees well with the transient result. The 5 harmonics solution does not show further improvement; therefore the number of harmonics higher than $n=3$ is not needed in this case. As presented in Fig. 9 linear approximation of mesh flux, described in the Mesh Motion section, does not introduce significant errors for cases with high velocities and small amplitude mesh motions.

Pressure contours for the transient and 5 harmonics solution near the airfoil is presented in Fig. 10. Overall flow fields exhibit similar behaviour.

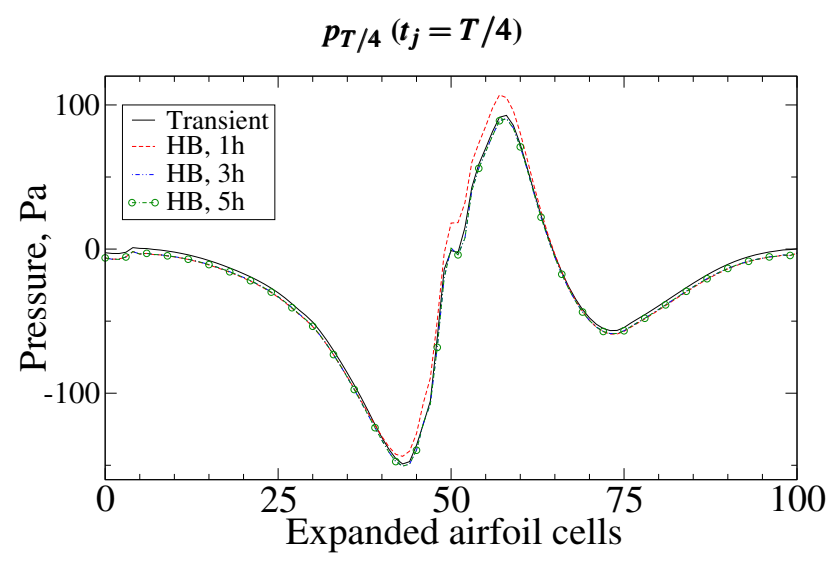

FIGURE 8: COMPARISON OF PRESSURE CONTOURS AROUND THE AIRFOIL AT HIGH $R e$ FOR $t_{j}=T / 4$. $p_{2 T / 4}\left(t_{j}=2 T / 4\right)$
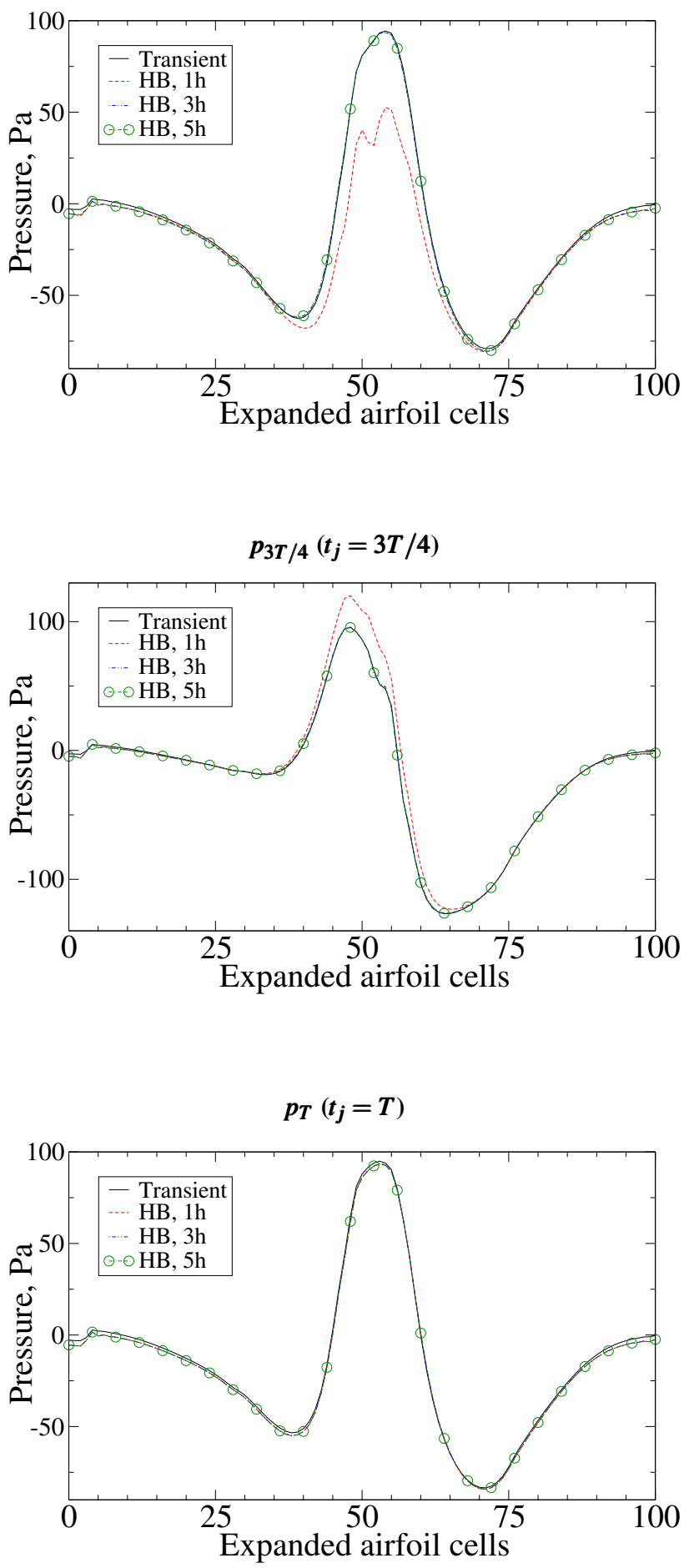

FIGURE 9: PRESSURE CONTOURS AROUND THE AIRFOIL AT HIGH $R e$ FOR $t_{j}=2 T / 4,3 T / 4$ and $T$. 


\section{Transient simulation}

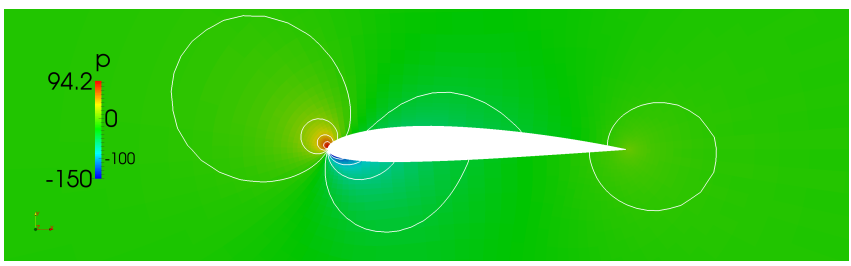

Harmonic balance, 5 harmonics

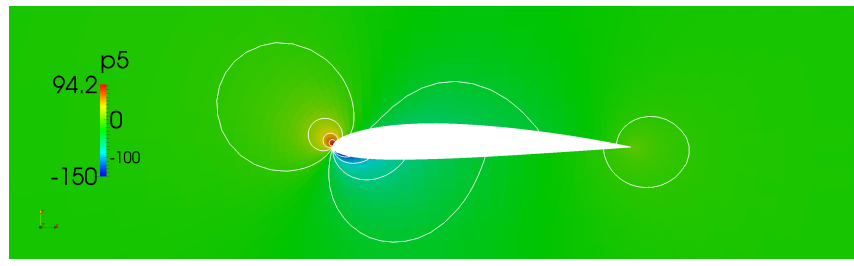

FIGURE 10: HB AND TRANSIENT SIMULATION PRESSURE FIELD AT $t_{j}=T / 4$.

Pitching Onera M6. Onera M6 is used to demonstrate HB performance for 3D problems. The domain is cylindrical, discretised with 728399 cells, depicted in Fig. 11. Inlet velocity is set to $5 \mathrm{~m} / \mathrm{s}$ and kinematic viscosity is $v=1.5 \cdot 10^{-5} \mathrm{~m}^{2} / \mathrm{s}$. Pitching angle amplitude is $3 \mathrm{deg}$, following the sine law. Axis of pitching is located at wing chord, one fifth distance from the leading edge. Pitching period is set to $10 \mathrm{~s}$.

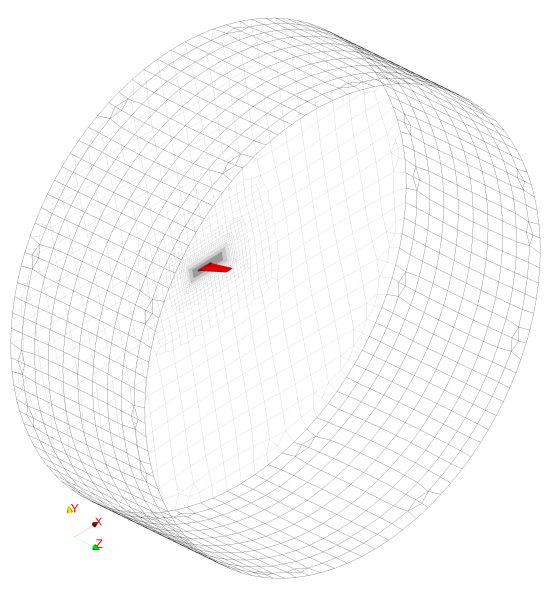

FIGURE 11: ONERA M6 DOMAIN, WING IS COLOURED RED.

With presented insignificant differences between 3 and 5 harmonics in NACA 2412 cases, Onera M6 test case is run us- ing only 1 and 3 harmonics. Comparison is carried out at three cross-sections: at 0.1 wing span, 0.5 wing span and 0.9 wing span. For all three cross-sections, HB results are compared to transient ones in four time instants $t=T / 4, t=2 T / 4, t=3 T / 4$ and $t=T . \quad$ Comparison is presented in Figures 12 to 17 showing good agreement between results. Looking at the pressure peak, it can be noticed that the periodic motion is successfully captured. All of the presented cross-sections exhibit the same accuracy for both 1 and 3 harmonics. 1 harmonic solution overlaps with the 3 harmonics solution, except in the region of pressure peak where 3 harmonics demonstrate superior results.

The simulation was run in parallel using four cores on an Intel Core I5-3570K, $3.4 \mathrm{GHz}$ computer. The significant CPU time reduction from transient to $\mathrm{HB}$ simulation can be noticed: one period of transient simulation took $\sim 20$ hours of CPU time, while HB simulation with 1 harmonic took $\sim 4$ hours and nearly 1000 iterations. The 3 harmonics HB simulation took substantially longer, $\sim 15$ hours CPU time, converging in approximately 1000 iterations. Transient simulation time step was set to $\Delta t=5 \cdot 10^{-4} \mathrm{~s}$. It is unclear how many periods it would take for transient simulation to reach periodic steady state as the simulation was initialized with converged HB flow field. Usually a number of periods have to be run before reaching fully periodic steady state, meaning that CPU time of 1 period should be multiplied.
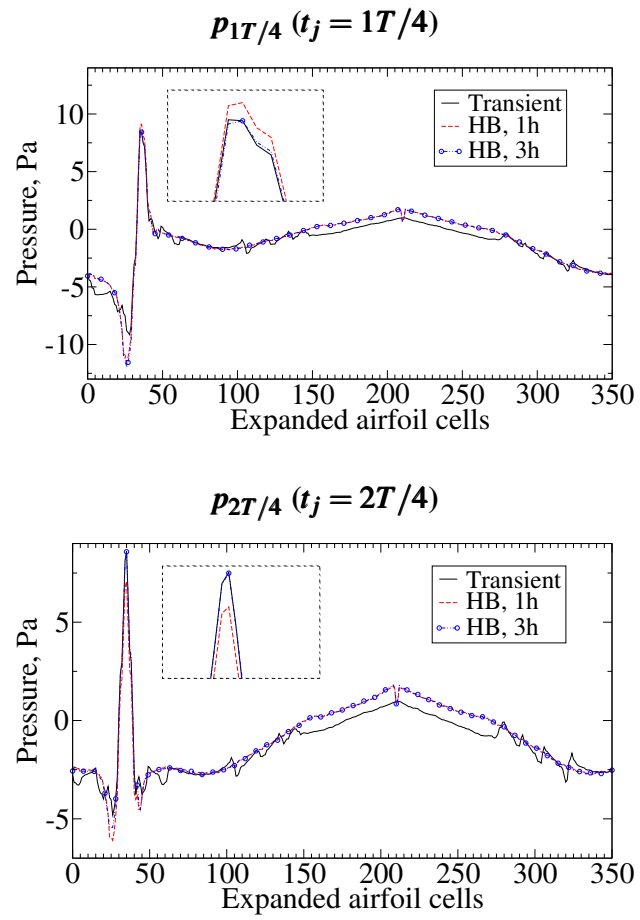

FIGURE 12: PRESSURE CONTOUR AT 0.1 WING SPAN FOR $t_{j}=T / 4$ and $2 T / 4$. 

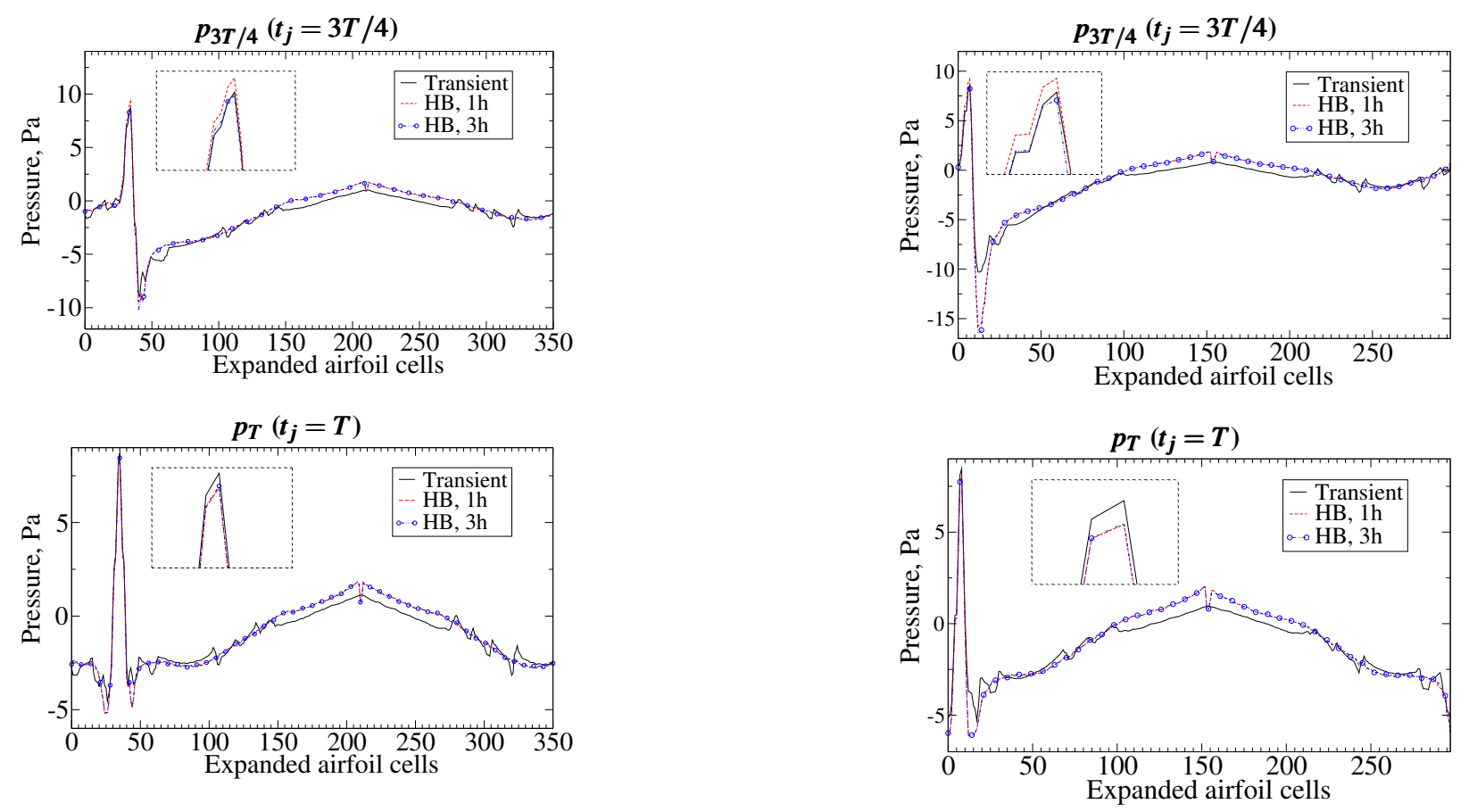

FIGURE 13: PRESSURE CONTOUR AT 0.1 WING SPAN FOR $t_{j}=3 T / 4$ and $T$.
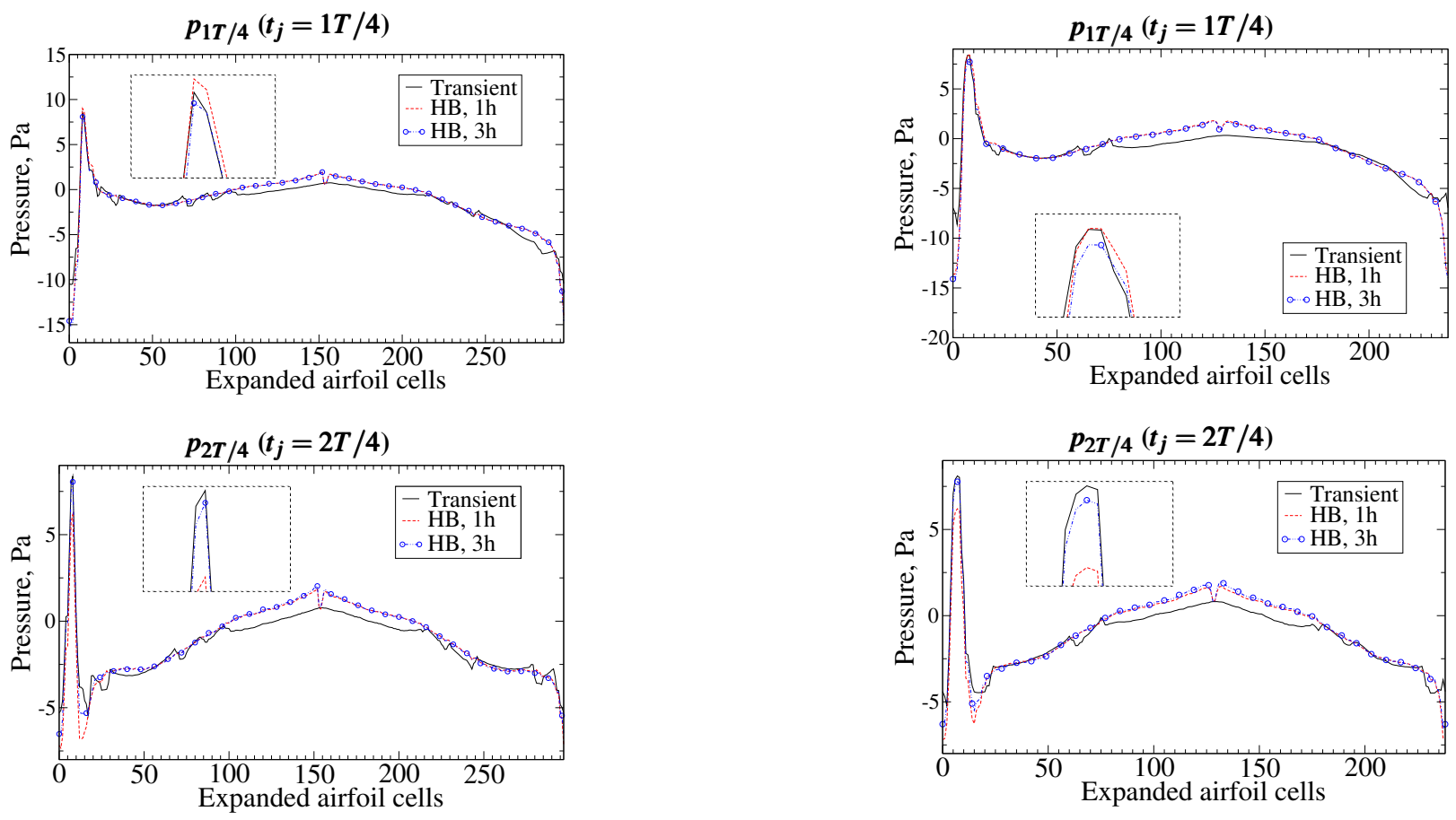

FIGURE 14: PRESSURE CONTOUR AT 0.5 WING SPAN FOR $t_{j}=T / 4$ and $2 T / 4$.

FIGURE 15: PRESSURE CONTOUR AT 0.5 WING SPAN FOR $t_{j}=3 T / 4$ and $T$.

FIGURE 16: PRESSURE CONTOUR AT 0.9 WING SPAN FOR $t_{j}=T / 4$ and $2 T / 4$. 

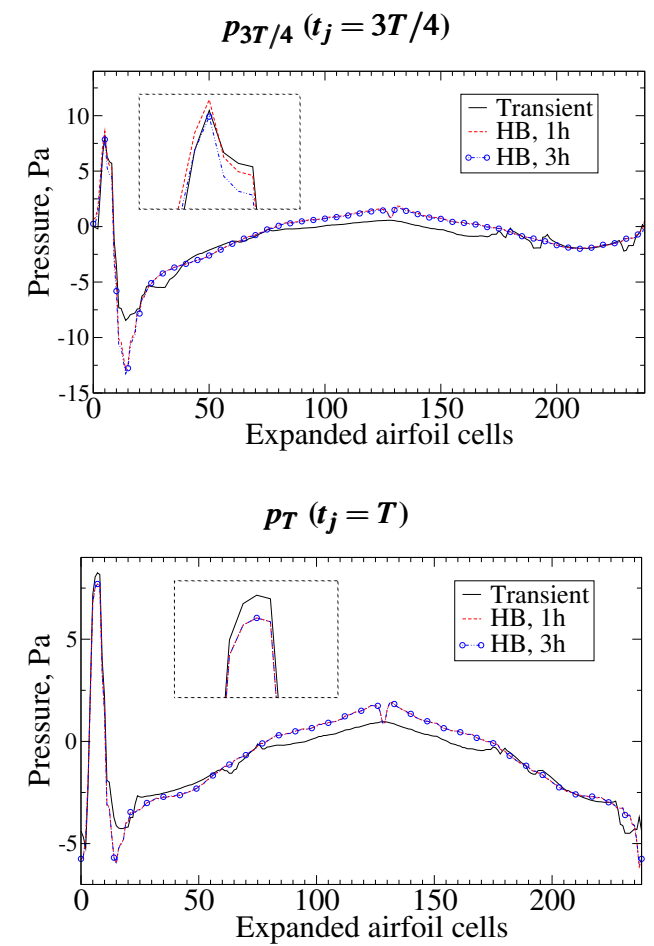

FIGURE 17: PRESSURE CONTOUR AT 0.9 WING SPAN FOR $t_{j}=3 T / 4$ and $T$.

\section{CONCLUSION}

Harmonic balance method for periodic problems is presented in this paper. HB is an efficient method for unsteady periodic flows with prescribed motion. Using the Fourier decomposition, a set of steady state equations is obtained. Time-derivative term is transformed into a source term, acting as a temporal coupling term in $2 n+1$ steady state equations. This approach should reduce simulation time with a negligible decrease in accuracy.

Validation and verification of the method is presented using four cases for scalar transport and three cases for incompressible Navier-Stokes equations. As a preliminary means of validation, scalar transport is considered first. Two cases demonstrated the HB performance with imposed harmonic waves, while the other two cases demonstrated the HB robustness with imposed steep waves. Incompressible turbulent flow is validated using pitching 2D airfoil with high and low Re setup and 3D wing. Good agreement is achieved in all of the cases. Compared to low $R e$ case, discrepancies between HB and transient solution are smaller in the high $R e$ case.

Presented cases demonstrate the HB accuracy and efficiency compared to conventional transient solvers. Comparison of pressure contours shows that flow features around the airfoil (wing) are successfully captured. Only the pitching cases were shown here, as they include small mesh motions. At this point, the capability for simulating rotating machinery is under development and it will be a topic of future publications. With presented test cases, showing that HB moving mesh approach yields accurate results, it is clear that this approach can be used for more complex problems such as turbines, propellers, etc., yielding significant CPU time savings.

\section{REFERENCES}

[1] Hall, K. C., Thomas, J. P., and Clark, W. S., 2002. "Computation of unsteady nonlinear flows in cascades using a harmonic balance technique". AIAA Journal, 50(5), pp. 879886.

[2] Chen, T., Vasanthakumar, P., and He, L., 2001. "Analysis of unsteady blade row interaction using nonlinear harmonic approach". Journal of propulsion and power, 17(3), pp. 651-658.

[3] Cvijetić, G., Jasak, H., and Vukević, V., 2015. "Finite volume implementation of harmonic balance method for periodic non-linear flows". AIAA.

[4] He, L., 1992. "Method of simulating unsteady turbomachinery flows with multiple perturbations". AIAA Journal, 30, 11.

[5] He, L.; Ning, W., 1998. "Efficient approach for analysis of unsteady viscous flows in turbomachines". AIAA Journal, 36, 11.

[6] Dufour, G., Sicot, F., Puigt, G., Liauzun, C., and A., D., 2010. "Contrasting the harmonic balance and linearized methods for oscillating-flap simulations". AIAA Journal, 48(4).

[7] Thomas, J. P., Dowell, E. H., Hall, K. C., and Denegri Jr., C. M., 2005. "Further investigation of modeling limit cycle oscillation behavior of the $\mathrm{f}-16$ fighter using a harmonic balance approach". AIAA.

[8] Thomas, J. P., Custer, C. H., Dowell, E. H., and Hall, K. C., 2009. "Unsteady flow computation using a harmonic balance approach implemented about the overflow 2 flow solver". 19th AIAA Computational Fluid Dynamics.

[9] Ekici, K., and Hall, K. C., 2011. "Harmonic balance analysis of limit cycle oscillations in turbomachinery". AIAA Journal, 49(7).

[10] Sicot, F., Gomar, A., Dufour, G., and A., D., 2014. "Time-domain harmonic balance method for turbomachinery aeroelasticity". AIAA Journal, 52(1).

[11] Ekici, K., Thomas, J. P., Hall, K. C., and Voytovych, D. M., 2003. "Frequency domain techniques for complex and nonlinear flows in turbomachinery". 33rd AIAA Fluid Dynamics Conference and Exhibit.

[12] Nadarajah, S. K., and Jameson, A., 2007. "Optimum shape design for unsteady three-dimensinal viscous flows using a 
nonlinear frequency-domain method". Journal of Aircraft, 44(5).

[13] Gopinath, A. K., Weide, E. Alonso, J. J., A., J., Ekici, K., and Hall, K. C., 2007. "Three-dimensional unsteady multistage turbomachinery simulations using the harmonic balance technique". Collection of Technical Papers - 45th AIAA Aerospace Sciences Meeting, 16.

[14] Guédeney, T., Gomar, A., and Sicot, F., 2013. "Multifrequential harmonic balance approach for the computation of unsteadiness in multi-stage turbomachines". 2léme Congrés Français de Mécanique, Bordeaux.

[15] Hall, K., Ekici, K., Thomas, J., and Dowell, E., 2013. "Harmonic balance methods applied to computational fluid dynamics problems". International Journal of Computational Fluid Dynamics, 27(2), pp. 52-67.

[16] Rusche, H., 2002. "Computational fluid dynamics of dispersed two - phase flows at high phase fractions". $\mathrm{PhD}$ thesis, Imperial College of Science, Technology \& Medicine, London.

[17] Jasak, H., 1996. "Error analysis and estimation for the finite volume method with applications to fluid flows". PhD thesis, Imperial College of Science, Technology \& Medicine, London.

[18] Patankar, S. V., and Spalding, D. B., 1972. "A calculation procedure for heat, mass and momentum transfer in threedimensional parabolic flows". Int. J. Heat Mass Transf., 15(10), pp. 1787-1806.

[19] Saad, Y., 2003. Iterative Methods for Sparse Linear Systems. Society for Industrial and Applied Mathematics, SIAM.

[20] Menter, F. R., Kuntz, M., and Langtry, R., 2003. Ten Years of Industrial Experience with the SST Turbulence Model. Begell House, Inc.

[21] Demirdžić, I., and Perić, M., 1998. "Space conservation law in finite volume calculations of fluid flow". Int. J. Numer. Meth. Fluids, 8, pp. 1037-1050. 